\title{
Inflatable soft jumper inspired by shell snapping
}

\author{
Benjamin Gorissen $^{\mathrm{a},{ }^{*}, \text { David Melancon }^{\mathrm{a},{ }^{*}}, \text { Nikolaos Vasios }^{\mathrm{a}}, \text { Mehdi Torbati }^{\mathrm{a}} \text {, and Katia Bertoldi }}{ }^{\mathrm{a}, \mathrm{b}, \mathrm{c}, \dagger}$ \\ a J.A. Paulson School of Engineering and Applied Sciences, Harvard University, Cambridge, MA 02138, USA; b Wyss Institute for Biologically Inspired Engineering Harvard \\ University, Cambridge, MA 02138, USA; ' Kavli Institute for Bionano Science and Technology, Harvard University, Cambridge, MA 02138, USA
}

\begin{abstract}
Fluidic soft actuators are enlarging the robotics toolbox by providing flexible elements that can display highly complex deformations. While these actuators are adaptable and inherently safe, their actuation speed is typically slow as the influx of fluid is limited by viscous forces. To overcome this limitation and realize soft actuators capable of rapid movements, we focus on spherical caps that exhibit isochoric snapping when pressurized under volume-controlled conditions. First, we note that this snap-through instability leads to both a sudden release of energy and a fast cap displacement. Inspired by these findings, we investigate the response of actuators that comprise such spherical caps as building blocks and observe the same isochoric snapping mechanism upon inflation. Finally, we demonstrate that this instability can be exploited to make these actuators jump even when inflated at a slow rate. As such, our study provides the foundation for the design of a new class of fluidic soft devices that can convert a slow input signal into a fast output deformation.
\end{abstract}

Soft robots | Inflatable actuators | Isochoric snap-through | Energy release | Jumping

Inflatable soft actuators have emerged as an ideal platform to realize active structures capable of safe interactions with unstructured environments (1-3). Their compliance and ability to achieve complex deformations has enabled the design of flexible machines for a wide spectrum of applications (4), ranging from minimally invasive surgical tools (5) and exoskeletons (6) to warehouse grippers (7) and add-ons for video games (8). However, existing fluidic soft actuators are typically slow, since a significant amount of fluid has to be supplied for their operation, the influx of which is restricted by viscous forces in tubes and valves. To overcome this limitation, it has been shown that fast actuation can be achieved either by modifying the geometry to reduce the amount of fluid needed for inflation (9) or by using chemical reactions to generate explosive bursts of pressure (10). Alternatively, snapping instabilities can also provide a powerful nonlinear mechanism that decouples the slow input signal from the output deformation and triggers rapid events (11-15).

Inspired by recent progress using snapping instabilities to increase the speed of actuation $(11,16)$, we investigate the snapping of spherical caps as a mechanism to realize fluidic soft actuators capable of rapid movements. We first show that the snapping of elastomeric spherical caps upon pressurization results in a sudden release of energy, the amount of which can be controlled by tuning the caps' geometry, material stiffness, and boundary conditions at the edges. We then realize fluidic soft actuators by combining two spherical caps (see Fig. 1A) and find that the energy released upon snapping of the inner cap leads to a rapid inversion of its pole that ultimately enables jumping. Finally, we identify geometric and material parameters that result in significant energy release and jump height, providing a rich design domain for fluidic soft actuators capable of extremely fast movements regardless of inflation rate.

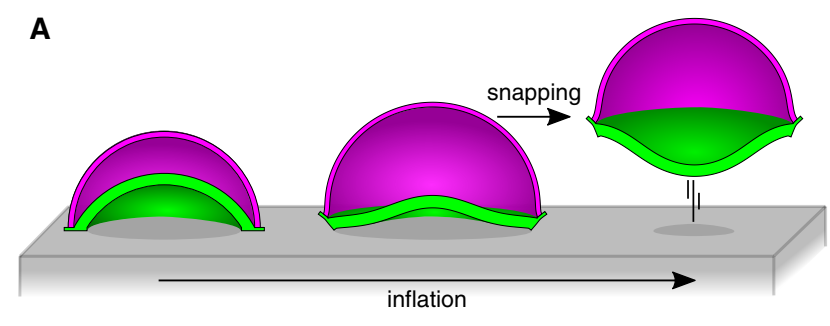

B

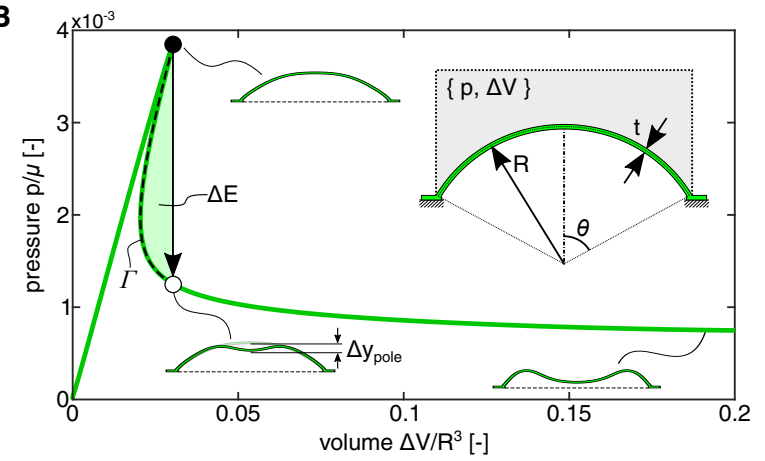

C

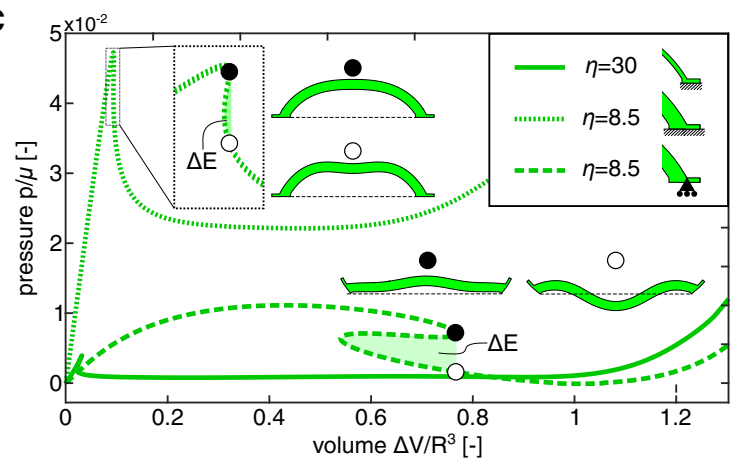

Fig. 1. Snapping of spherical caps for fast fluidic soft robots. A. Our soft fluidic actuators comprise two spherical caps connected at their base. Upon inflation the inner cap snaps and enables our simple device to take off. B. The pressure-volume curve, normalized by initial shear modulus $\mu$ and radius $R$, of a given pressurized spherical cap is characterized by a limit point when inflating under volume-controlled conditions. This volume limit point causes an isochoric snapping instability, which leads to a sudden release of energy, $\Delta E$ (highlighted in green), and the inversion of the inner cap (which we characterize by quantifying the distance travelled be the cap's pole, $\Delta y_{\text {pole }}$ ). C. Comparison between the pressure-volume curves of thin (solid green line) and thick spherical caps with both clamped (dotted green line) and roller (dashed green line) boundary conditions. Note that the normalized radius $\eta$ is defined as the ratio of cap radius over cap thickness (i.e. $\eta=R / t$ ). 


\section{Results}

Snapping of spherical caps as a platform for fast fluidic soft robots. To create fast inflatable soft actuators, we start by conducting Finite Element (FE) analyses to investigate the response upon pressurization of elastomeric spherical caps with radius, $R$, thickness, $t$, and polar angle, $\theta$ (see Fig. 1B). In our simulations (which are conducted using the commercial package ABAQUS 2018/Standard), we assume the deformation to be axisymmetric, discretize the models with 4-node bilinear axisymmetric solid elements, and use an incompressible Gent material model with initial shear modulus, $\mu$ (17). We pressurize the caps by supplying incompressible fluid to a cavity above them (highlighted in gray in the inset in Fig. 1B) and simulate the quasi-static pressure-volume curve via the modified Riks algorithm $(18,19)$. In Fig. 1B, we consider a thin cap with polar angle $\theta=60^{\circ}$, normalized radius $\eta=R / t=30$, and clamped boundary conditions at the base. We find that the pressure-volume curve of this cap is qualitatively identical to those recently reported for pressurized spherical shells (20-22) and is characterized by a limit point when inflating under volume-controlled conditions (indicated by a black circular marker in Fig. 1B). This volume limit causes an instability leading the system to snap to a point of lower pressure (white circular marker in Fig. 1B). This occurs while conserving the volume in the cavity and results in a partial inversion of the cap, which we characterize by quantifying the distance traveled by the pole during the instability, $\Delta y_{\text {pole }}$ (see insets in Fig. 1B). Further, we note that the isochoric snapping of the cap is accompanied by a sudden release of energy, $\Delta E$, that graphically corresponds to the green highlighted area in Fig. $1 \mathrm{~B}$ and can be obtained as

$$
\Delta E=\int_{\Gamma} p d \Delta V
$$

where $\Gamma$ is the equilibrium path that connects the limit point in volume and the corresponding isochoric point on the lower branch (highlighted by a dashed black line in Fig. 1B). For the considered cap, we find $\Delta E=1.50 \times 10^{-5} \mu R^{3}$ and $\Delta y_{\text {pole }}=1.31 \times 10^{-1} R$.

Next, to investigate the effect of geometry on $\Delta E$ and $\Delta y_{\text {pole }}$, we compare the response of the thin spherical cap to that of a thicker one with $\eta=8.5$ and both with clamped and roller boundary conditions at the base. The results reported in Fig. $1 \mathrm{C}$ indicate that the boundary conditions play a major role on the snapping behavior. The clamped thick cap is characterized by a very large maximum pressure, but a very small energy release and pole displacement upon snapping $\left(\Delta E=4.78 \times 10^{-6} \mu R^{3}\right.$ and $\left.\Delta y_{\text {pole }}=6.30 \times 10^{-2} R\right)$. On the other hand, roller boundary conditions lower the maximum pressure, but lead to a much higher energy release and pole displacement $\left(\Delta E=8.00 \times 10^{-4} \mu R^{3}\right.$ and $\left.\Delta y_{\text {pole }}=2.67 \times 10^{-1} R\right)$. As a result, our simulations indicate not only that isochoric snapping of spherical caps provides opportunities to realize systems capable of suddenly releasing a significant amount of energy through their inversion, but also that by tuning geometry and boundary conditions we can control and maximize the response of these systems.
Inflatable soft actuators inspired by shell snapping. Having demonstrated numerically that snapping of a spherical cap results in a sudden energy release and pole displacement, we now investigate the mechanical response of fully soft actuators comprising two spherical caps connected at their base to form a cavity that we inflate with an incompressible fluid (see Fig. 1A). We begin by considering three actuators with inner caps identical to those introduced in Fig. $1 \mathrm{C}$ (with $R_{i}=30 \mathrm{~mm}$ ) with outer caps characterized by $\theta_{o}=90^{\circ}$ and $\eta_{o}=16.5$ (note that here and in the following the subscripts $o$ and $i$ are used to indicate properties of the outer and inner caps, respectively). Specifically, Design A has a thin inner cap with $\theta_{i}=60^{\circ}$ and $\eta_{i}=30$, while Designs B and $\mathrm{C}$ have a thick cap with $\theta_{i}=60^{\circ}$ and $\eta_{i}=8.5$. Further, while we assume that both caps of Designs A and B are made of the same elastomeric material (i.e. $\mu_{i} / \mu_{o}=1$ ), for Design $\mathrm{C}$ we consider an outer cap made of a softer rubber, resulting in $\mu_{i} / \mu_{o}=5.8$. The numerically obtained pressure-volume curves for the three actuators (shown in Fig. 2A as blue lines) share many features with those reported in Fig. 1C for the individual pressurized caps and are all characterized by a limit point near to the maximum pressure when considering volume-controlled conditions. As such, our actuators also exhibit isochoric snapping upon inflation and this again results in a sudden release of elastic energy and the inversion of the inner cap. By comparing the responses of the three actuators, we find that Design C exhibits the largest energy release and displacement of the inner cap's pole $(\Delta E=0.875 \mathrm{~mJ}, 5.67 \mathrm{~mJ}$, and $31.4 \mathrm{~mJ}$ for Designs A, B and $\mathrm{C}$, respectively, whereas $\Delta y_{\text {pole }}=7.68 \mathrm{~mm}, 7.55 \mathrm{~mm}$, and $20.89 \mathrm{~mm}$ for Designs A, B, and C, respectively). These results agree with the trends observed for the individual pressurized caps (see Fig. 1C), as both $\Delta E$ and $\Delta y_{\text {pole }}$ increase for thicker caps that are allowed to rotate at their base (note that in our actuators such boundary conditions are not directly controlled, but rather determined by the outer cap - see Fig. S13). Furthermore, the results highlight the important role of the outer caps and indicate that both $\Delta E$ and $\Delta y_{\text {pole }}$ can be enhanced by increasing their compliance. This is because compliant outer caps can sustain more deformation prior to snapping (see insets in Fig. 2A) and, therefore, enable the actuator to store more elastic energy that can be potentially released upon instability.

Next, to experimentally validate our analyses, we fabricate the three actuators using molds and inflate them with water while submerged in water to eliminate the effects of gravity (see Supplementary Materials for details). In this experimental analysis, all caps are fabricated out of Zhermack Elite Double 32 (with green color and initial shear modulus $\mu=0.35 \mathrm{MPa}$ ), except for the outer cap of Design $\mathrm{C}$ where we use Zhermack Elite Double 8 (with purple color and initial shear modulus $\mu=0.06 \mathrm{MPa}$ ). Note that these values are obtained by minimizing the error between experiments and simulations for Design $\mathrm{C}$ and are within the range previously reported in the literature $(21,23-25)$. In Fig. 2A, we compare the numerical (blue lines) and experimental (red lines) pressure-volume curves for the three actuators, while in Fig. $2 \mathrm{~B}$ we display snapshots that are taken during the tests. We find good agreement between simulations and experiments, with pressure measurements that show a sudden drop near the numerically predicted limit point. The small discrepancies between experiments and simulations can be attributed 

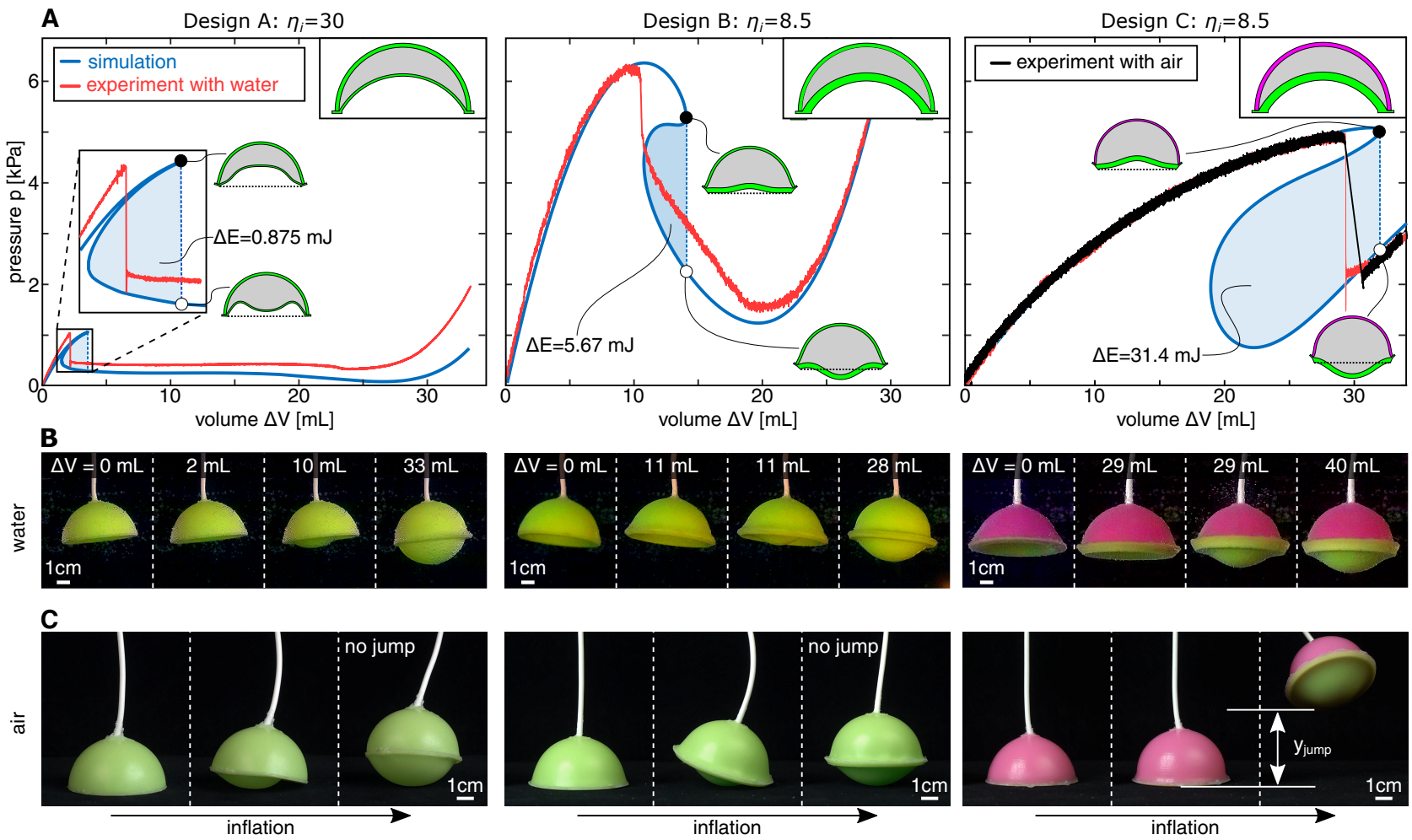

Fig. 2. Our inflatable soft actuators. A Experimental (red) and numerical (blue) pressure-volume curves for the inflation with water of three actuators (all with inner cap radius $\left.R_{i}=30 \mathrm{~mm}\right)$ characterized by normalized radii, polar angles, and ratio of shear moduli $\left(\eta_{i}, \theta_{i}, \eta_{o}, \theta_{o}, \mu_{i} / \mu_{o}\right)=\left(30,60^{\circ}, 16.5,90^{\circ}, 1\right)($ Design $\mathrm{A})$, $\left(8.5,60^{\circ}, 16.5,90^{\circ}, 1\right)$ (Design $\left.\mathrm{B}\right)$, and $\left(16.5,60^{\circ}, 16.5,90^{\circ}, 5.8\right)$ (Design $\mathrm{C}$ ). The energy released upon snapping, $\Delta E$, is highlighted by the shaded blue region. The volume limit point upon inflation is identified with a black circular marker, while its corresponding isochoric point on the lower branch is identified with a white circular marker. For Design C, we also report the experimental pressure-volume curve obtained when inflating the actuator with air (black line). B Experimental snapshots of the three design during inflation with water at different amounts of supplied fluid. $\mathbf{C}$ Experimental snapshots of the three designs during inflation with air. The isochoric snapping makes Design $\mathrm{C}$ jump and reach a maximum height denoted $y_{j u m p}$. 

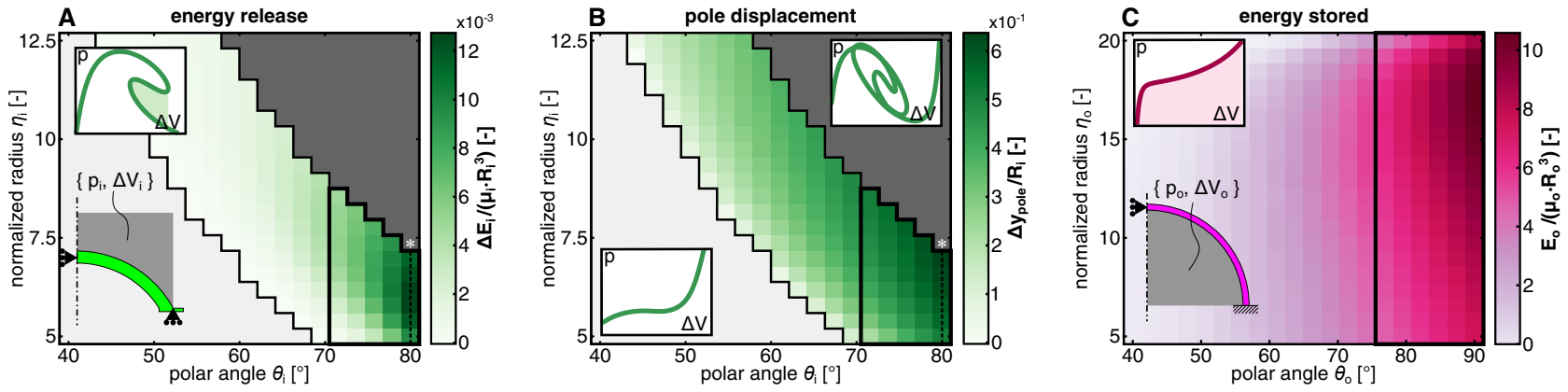

Fig. 3. Mechanical response of the inner and outer caps upon inflation. (A)-(B) Evolution of the inner cap's normalized $(\mathbf{A})$ energy release, $\Delta E_{i} /\left(\mu_{i} R_{i}^{3}\right)$, and $(\mathbf{B})$ pole displacement, $\Delta y_{\text {pole }} / R_{i}$, upon snapping as a function of the normalized radius, $\eta_{i}$, and the polar angle, $\theta_{i}$. $\mathbf{C}$. Evolution of the outer cap's normalized stored energy at $p / \mu_{o}=0.5, E_{o} /\left(\mu_{o} R_{o}^{3}\right)$, as a function of the normalized radius, $\eta_{o}$, and the polar angle, $\theta_{o}$.

to unavoidable imperfections introduced during fabrication, visco-elasticity of the rubber, and slight asymmetric buckling of the inner cap. Furthermore, in all of our tests, as observed in the simulations, snapping is also accompanied by the sudden inversion of the inner cap. While $\Delta y_{\text {pole }}$ for Design A is such that the inner cap's pole remains above the base plane of the actuator upon snapping, for Designs B and C the large value of $\Delta y_{\text {pole }}$ allows for their inner cap's pole to cross it (see Fig. 2B).

Motivated by these results, we investigate how snapping can be exploited to enhance the functionality of our simple robots and make them jump even when inflated at a slow rate. To this end, we position our actuators on a flat surface and slowly inflate them with air (see Supplementary Materials for details). The snapshots reported in Fig. $2 \mathrm{C}$ reveal that, despite the slow rate of inflation $(10 \mathrm{~mL} / \mathrm{min}$ with a syringe pump), the isochoric snapping makes Design C jump and reach a maximum height of $y_{j u m p}=42.9 \mathrm{~mm}$. On the other hand, even though the inner cap of Designs A and B snaps upon inflation, their $\Delta E$ and $\Delta y_{\text {pole }}$ are not large enough to enable them to take off. Although this last set of test was conducted using a compressible fluid (air), the effect of fluid compressibility on the response of the system energy release is negligible and only leads to a slight increase of volume during snap-through (see Fig. 2A and Supplementary Materials for details). As such, the experiments and analyses conducted using an incompressible fluid can be also used to understand and improve the performance of our air-inflated jumpers.

Improving the actuators' response. Thus far we have shown that the geometry and material properties of the caps strongly affect the snapping behavior and that by tuning $\Delta E$ and $\Delta y_{\text {pole }}$ we can harness the instability to make our actuators jump. Motivated by these findings, we proceed by systematically exploring the parameter space to identify designs that can jump higher than Design C. Fig. 2C indicates that jumping requires large enough $\Delta E$ and $\Delta y_{\text {pole }}$ and Fig. $2 \mathrm{~A}$ that $\Delta E$ and $\Delta y_{\text {pole }}$ can be enhanced by combining an inner cap that releases a large amount of energy upon snapping with an outer cap that stores a large amount of energy prior to snapping. Therefore, we start by considering the two caps separately and use axisymmetric FE analyses to investigate their behavior for a wide range of geometric parameters (i.e. $40^{\circ} \leq \theta_{i} \leq 80^{\circ}$, $\left.5 \leq \eta_{i} \leq 12.5,40^{\circ} \leq \theta_{o} \leq 90^{\circ}, 5 \leq \eta_{o} \leq 20\right)$.

Focusing on the inner cap, we find that by varying $\theta_{i}$ and $\eta_{i}$ its response undergoes several transitions (see Fig. 3A-B). For low values of $\theta_{i}$ and $\eta_{i}$ (i.e. for thick and shallow caps), the inner cap does not exhibit isochoric snapping (see light gray region in Fig. 3A-B). By increasing $\theta_{i}$ at constant $\eta_{i}$, snapping is eventually triggered upon inflation. Within this domain both the energy released by the inner cap, $\Delta E_{i}$, and its pole displacement, $\Delta y_{\text {pole }}$, increase monotonically as a function of $\theta_{i}$, suggesting that the response of our actuators can be enhanced by considering deep and sufficiently thick inner caps. Finally, for high values of $\theta_{i}$ and $\eta_{i}$ (i.e. for thin and deep caps) the pressure-volume curves become self-crossing (see dark gray region in Fig. 3A-B), which is an indication of the existence of a more favorable asymmetric deformation path with low $\Delta E_{i}$ and $\Delta y_{\text {pole }}$ (see Fig. S16).

Next, we turn our attention to the outer cap and find that its response is less rich and resembles that of an inflated thin spherical balloon $(26,27)$. Since the outer cap in our actuators acts as an energy reservoir, in Fig. 3C, we report the evolution of the stored energy in the outer cap, $E_{o}$, at a normalized pressure of $p / \mu_{o}=0.5$, as a function of the polar angle $\theta_{o}$ and the normalized radius $\eta_{o}$. The results indicate that $E_{o}$ increases monotonically with $\theta_{o}$ (almost irrespective of $\eta_{o}$ ), therefore suggesting that the response of our actuators can be enhanced by focusing on deep outer caps.

While the results of Fig. 3 enable us to identify promising regions of the design space (i.e. inner caps with $\theta_{i} \geq 70^{\circ}$ and $\eta_{i} \leq 8$ and outer ones with $\theta_{o} \geq 76^{\circ}$ ), they cannot be directly used to realize the best possible jumper as they neglect the coupling between the two caps. Therefore, as next step, we use axisymmetric FE analyses to simulate the response of 4800 actuators constructed by combining inner and outer caps within the identified promising regions (highlighted by black contours in Fig. 3). In Fig. $4 \mathrm{~A}$, we report $\Delta E$ and $\Delta y_{\text {pole }}$ for all simulated actuators with both $\mu_{i} / \mu_{o}=1$ (green markers) and 5.8 (purple markers). Four key features emerge from the plot. First, by comparing the results with those obtained for the three actuators considered in Fig. 2 (indicated by square markers in Fig. 4A) we find that both $\Delta E$ and $\Delta y_{\text {pole }}$ can be greatly increased when the geometry is properly tuned. Second, the results show that, on one hand, there is a strong correlation between $\Delta E$ and $\Delta y_{\text {pole }}$ and, on the other hand, there is a disconnection between them and the drop in pressure associated with the snapping instability. Specifically, by inspecting the pressure-volume curves for the actuators (shown as insets in Fig. 4A) we find that for the designs with large $\Delta E$ and $\Delta y_{\text {pole }}$ the drop in pressure is small, while the area enclosed by the pressure-volume curve 


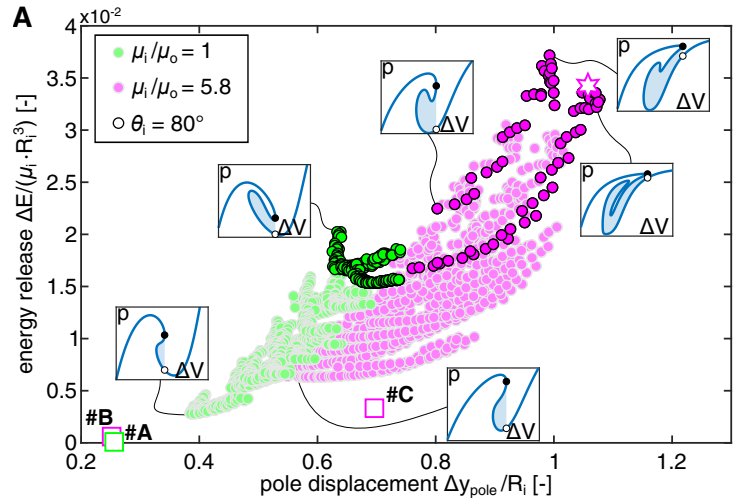

B
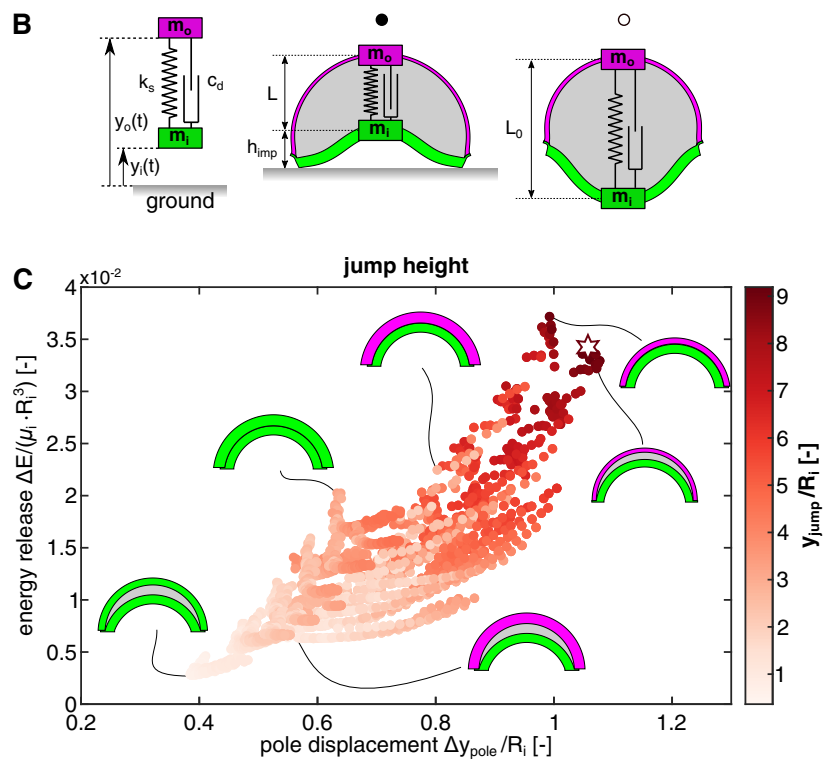

Fig. 4. Improving the response of the actuators. A. Normalized energy release, $\Delta E /\left(\mu_{i} R_{i}^{3}\right)$, vs. normalized pole displacement, $\Delta y_{\text {pole }} / R_{i}$, for actuators with inner polar angle $\theta_{i} \geq 70^{\circ}$, normalized inner radius $\eta_{i} \leq 8$, and normalized outer radius $\theta_{o} \geq 76^{\circ}$. B. Reduced order mass-spring model used to predict jump height based on the numerical results reported in $\mathbf{A}$. The model comprises two masses $m_{i}$ and $m_{o}$ connected via a spring with stiffness $k_{s}$ and a dashpot with damping coefficient $c_{d}$. C. Normalized jump height, $y_{j u m p} / R_{i}$ as a function of energy release and pole displacement for the 4800 actuators considered in $\mathbf{A}$.

between the limit point and the corresponding isochoric point on the lower branch is large. Third, the results confirm the importance of a flexible outer cap as both $\Delta E$ and $\Delta y_{\text {pole }}$ are significantly larger for the actuators with $\mu_{i} / \mu_{o}=5.8$. Fourth, we find that the inner cap plays a crucial role and that by choosing $\theta_{i}=80^{\circ}$ to optimize its response we significantly improve the performance of the actuators (see black contour markers in Fig. 4A). At the same time, however, the results also highlight that for actuators with $\mu_{i} / \mu_{o}=5.8$ the outer cap geometry is important, as some design choices lead to noticeably lower $\Delta E$ and $\Delta y_{\text {pole }}$.

Our quasi-static FE simulations allow us to efficiently explore the design space and calculate $\Delta E$ and $\Delta y_{\text {pole }}$ for a large number of designs. However, since they do not account for dynamic effects, they cannot be used to directly characterize the ability of the actuators to jump. To overcome this limitation, we establish a simple mass-spring model that takes the FE results of Fig. 4A as input and predicts the jump height, $y_{\text {jump }}$. This reduced order model comprises two concentrated masses, $m_{i}$ and $m_{o}$, connected by a spring with stiffness $k_{s}$ and rest length $L_{0}$ (Fig. 4B). We choose $m_{i}$ and $m_{o}$ to be equal to the mass of the inner and outer cap, respectively, and to be located at their corresponding poles. We then focus on the numerically predicted configurations immediately before and after snapping and assume that the mechanical system stores an amount of energy equal to $\Delta E$ in the former and is stress-free in the latter. It follows that $L_{0}$ is equal to the distance between the poles immediately before snapping and that (see Fig. 4B)

$$
k_{s}=\frac{2 \Delta E}{\left(\Delta y_{\text {pole }}\right)^{2}} .
$$

Finally, we consider the spring to be initially pre-compressed by $\Delta y_{\text {pole }}$ and $m_{i}$ to be positioned at a distance $h_{i m p}$ from the ground ( $h_{i m p}$ being the numerically predicted distance of the inner cap's pole from the ground immediately before snapping). At time $t=0$, we release the system and analytically determine the position of the two masses, $y_{i}(t)$ and $y_{o}(t)$, as a function of time while accounting for contact with a rigid surface.

To verify the validity of our simplified massspring model, we first focus on three designs with $\left(\eta_{i}, \theta_{i}, \eta_{o}, \theta_{o}, \mu_{i} / \mu_{o}\right)=\left(8.5,60^{\circ}, 16.5,90^{\circ}, 5.8\right) \quad($ Design $\mathrm{C})$, $\left(5.4,80^{\circ}, 15.3,87^{\circ}, 5.8\right)$, and $\left(5.8,80^{\circ}, 10.5,85^{\circ}, 5.8\right)$ and compare the experimentally measured jump heights $\left(h_{\text {jump }}=42.9 \mathrm{~mm}, 160 \mathrm{~mm}\right.$, and $209 \mathrm{~mm}$, respectively) to the predicted ones. When choosing a coefficient of restitution $\alpha=0.5$ and approximating the effect of dissipation with a linear dashpot with damping coefficient $c_{d}=0.4 \mathrm{~kg} / \mathrm{s}$, we find excellent agreement between the two sets of data, with the model predicting $y_{\text {jump }}=\max \left(y_{i}(t)\right)=41.4 \mathrm{~mm}$, $175 \mathrm{~mm}$, and $226 \mathrm{~mm}$ (see Supplementary Materials for details). As such, these results indicate that our simple mass-spring model, despite the fact that it cannot capture the complex dynamic behavior typical of shells (14, 28, 29), can accurately predict the jump height of our soft jumpers. Having confirmed the validity of our model, we then use it to calculate $y_{\text {jump }}$ for all the 4800 actuators considered in Fig. 4A. The results reported in Fig. 4C clearly indicate

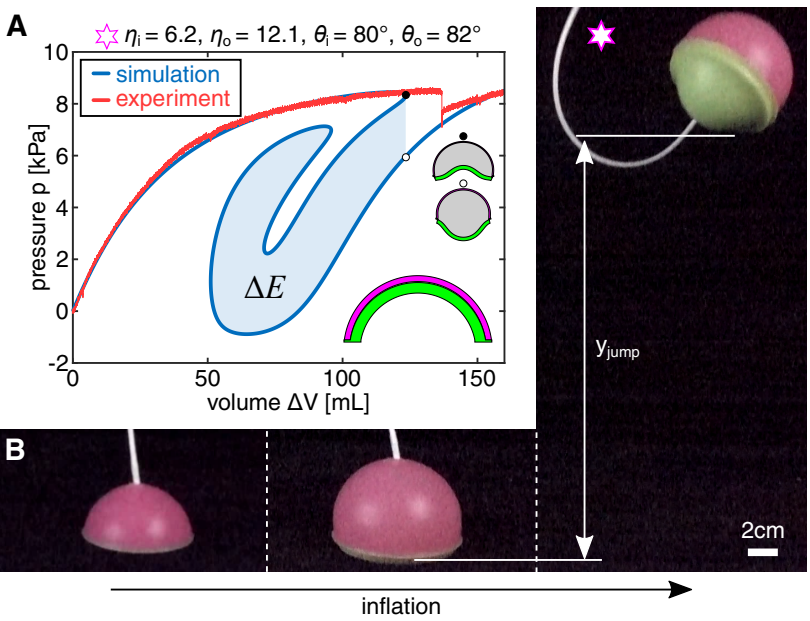

Fig. 5. Actuator with highest jump height. A. Numerical (blue line) and experimental (red line) pressure-volume for the actuator with normalized radii, polar angles, and ratio of shear moduli $\left(\eta_{i}, \theta_{i}, \eta_{o}, \theta_{o}, \mu_{i} / \mu_{o}\right)=\left(6.2,80^{\circ}, 12.1,82^{\circ}, 5.8\right)$ and inner cap radius $R_{i}=30 \mathrm{~mm}$. B Experimental snapshots of the actuator upon inflation with air. 
that a high jump requires both $\Delta E$ and $\Delta y_{\text {pole }}$ to be large. Specifically, we find that the jump height is highest for an actuator with $\left(\eta_{i}, \theta_{i}, \eta_{o}, \theta_{o}, \mu_{i} / \mu_{o}\right)=\left(6.2,80^{\circ}, 12.1,82^{\circ}, 5.8\right)$ for which $\Delta E=324 \mathrm{~mJ}$ and $\Delta y_{\text {pole }}=31.7 \mathrm{~mm}$. For such an actuator, our model predicts $y_{\text {jump }}=275 \mathrm{~mm}$, a jump height that is one order of magnitude larger than that previously recorded for Design C. Remarkably, our experimental results fully confirm the numerical predictions for this design for both the pressure-volume curve (see Fig. 5A) and the jump height $y_{\text {jump }}=283 \mathrm{~mm}$ (see Fig. $5 \mathrm{~B}$ ), further reinforcing the validity and efficiency of our numerical scheme to identify actuators with improved performance.

\section{Conclusion}

In summary, we have introduced a new family of inflatable soft actuators that harness isochoric snapping to move rapidly and even jump when inflated slowly. This remarkable behavior is encoded in their pressure-volume relationship, which exhibits two limit points in volume. While fluidic actuators are typically characterized by a monotonic pressure-volume curve $(9,30)$, it has been recently shown that limit points in pressure can be exploited to enhance their functionality and enable sequencing (31-33). Here, we show that by introducing limit points in volume we can realize soft robots capable of suddenly releasing a given amount of energy. Since the instability occurs at constant volume and does not involve transfer of fluid, the release of energy is extremely fast and enables us to convert the slow input signal into exceptionally rapid events such as jumps. Finally, our actuators can be simply reset and brought back to the initial configuration through vacuum and, therefore, can take off repetitively (see Movie S6).

Although in this study we have demonstrated the concept for spherical caps at the centimeter-scale, our approach can be extended to any shape and does not depend on size. Remarkably, since both $\Delta E$ and the gravitational potential energy are proportional to the mass, the jump height is independent of size (34). As such, we expect the relative jump, $y_{\text {jump }} / R_{i}$, to monotonically increase as the actuators are scaled down. On the other hand, on-board actuation and control may be embedded in larger jumpers (as the mass of these additional elements become negligible compared to that of the actuators) and open up the way to real-world applications requiring untethered soft robots $(35,36)$. Finally, while in this study we have focused on the response of spherical caps under inflation, similar highly non-linear behavior (i.e. force-displacement curves characterized by limit points in displacement) has been reported for the indentation of shallow arches (37) and shells with curved creases (38). Since structural elements with limit points in force have already been used to realize mechanical metamaterials with novel properties (39-43), we believe that by integrating these new building blocks into their design we can further expand their modes of functionality.

\section{Materials and Methods}

Details of the design, materials, and fabrication methods are summarized in Supplementary Materials, Sections S1 and S2. The experimental procedures, including the inflation with water to measure the pressure-volume curve and the inflation with air to measure jump height are described in Supplementary Materials, Section S3. FEA procedures and jumper mass-spring model are detailed in Supplementary Materials, Sections S4 and S5. Validation of the FEA model and jumper mass-spring model is reported in Supplementary Materials S6.

\section{Acknowledgments}

Funding: Research was supported by the NSF grants DMR1420570 and DMR-1922321 as well as the Fund for Scientific Research-Flanders (FWO). Author contributions: B.G. proposed the research idea, B.G., D.M., N.V., M.T. and K.B. developed the concept and designed the research, D.M. conducted the numerical simulations. B.G. fabricated the prototypes. B.G. and D.M. performed the experiments. B.G., D.M., and K.B. postprocessed the data and wrote the paper. K.B. supervised the research. Competing interests: The authors declare no conflict of interest. Data and materials availability: All data needed to evaluate the conclusions in the paper are present in the paper or the Supplementary Materials

\section{Supplementary materials}

Supplementary materials sections S1 to S6

Fig. S1. Ideal design.

Fig. S2. Actuator design.

Fig. S3. Fabrication.

Fig. S4. Our soft inflatable actuators.

Fig. S5. Inflation with water.

Fig. S6. Experimental pressure-volume curves of our actuators.

Fig. S7. Inflation with air.

Fig. S8. Jumping.

Fig. S9. Effect of air compressibility.

Fig. S10. Pressure-volume curves of our actuators.

Fig. S11. Pole displacement of our actuators.

Fig. S12. 3D simulations.

Fig. S13. Axisymmetric simulations of our actuators.

Fig. S14. Extracting $\Delta E$ from the numerical p-v curves.

Fig. S15. Axisymmetric simulations of the inner cap.

Fig. S16. Asymmetric deformation.

Fig. S17. Axisymmetric simulations of the outer cap.

Fig. S18. Mass-spring model to predict jump height.

Fig. S19. Jump height prediction.

Fig. S20. Validation of the spring-mass model.

Fig. S21. Improving the performance our soft fluidic actuators.

Fig. S22. Influence of the outer cap stiffness.

Movie S1. Snapping of spherical caps results in a sudden release of elastic energy.

Movie S2. Fast fluidic soft robots.

Movie S3. Isochoric snapping enables jumping.

Movie S4. Simplified mass-spring model to predict jump height.

Movie S5. Improving jumper design to increase jump height.

Movie S6. Repetitive jumping.

\section{References}

1. Daniela Rus and Michael T Tolley. Design, fabrication and control of soft robots. Nature, 521 (7553):467, 2015 .

2. Carmel Majidi. Soft robotics: a perspective - current trends and prospects for the future. Soft Robotics, 1(1):5-11, 2014

3. Deepak Trivedi, Christopher D Rahn, William M Kier, and lan D Walker. Soft robotics: Biological inspiration, state of the art, and future research. Applied bionics and biomechanics, 5(3): 99-117, 2008.

4. Benjamin Gorissen, Dominiek Reynaerts, Satoshi Konishi, Kazuhiro Yoshida, Joon-Wan Kim, and Michael De Volder. Elastic inflatable actuators for soft robotic applications. Advanced Materials, 29(43):1604977, 2017.

5. Mark Runciman, Ara Darzi, and George P Mylonas. Soft robotics in minimally invasive surgery. Soft robotics, 2019 .

6. Panagiotis Polygerinos, Zheng Wang, Kevin C Galloway, Robert J Wood, and Conor J Walsh Soft robotic glove for combined assistance and at-home rehabilitation. Robotics and $\mathrm{Au}$ tonomous Systems, 73:135-143, 2015.

7. Eric Brown, Nicholas Rodenberg, John Amend, Annan Mozeika, Erik Steltz, Mitchell R Zakin, Hod Lipson, and Heinrich M Jaeger. Universal robotic gripper based on the jamming of granular material. Proceedings of the National Academy of Sciences, 107(44):18809-18814, 2010.

8. J. Barreiros, H. Claure, B. Peele, O. Shapira, J. Spjut, D. Luebke, M. Jung, and R. Shepherd. Fluidic elastomer actuators for haptic interactions in virtual reality. IEEE Robotics and Automation Letters, 4(2):277-284, April 2019. ISSN 2377-3774. 
9. Bobak Mosadegh, Panagiotis Polygerinos, Christoph Keplinger, Sophia Wennstedt, Robert $F$ Shepherd, Unmukt Gupta, Jongmin Shim, Katia Bertoldi, Conor J Walsh, and George M Whitesides. Pneumatic networks for soft robotics that actuate rapidly. Advanced functional materials, 24(15):2163-2170, 2014.

10. Robert F Shepherd, Adam A Stokes, Jacob Freake, Jabulani Barber, Phillip W Snyder, Aaron D Mazzeo, Ludovico Cademartiri, Stephen A Morin, and George M Whitesides. Using explosions to power a soft robot. Angewandte Chemie International Edition, 52(10):28922896, 2013.

1. Johannes TB Overvelde, Tamara Kloek, Jonas JA D'haen, and Katia Bertoldi. Amplifying the response of soft actuators by harnessing snap-through instabilities. Proceedings of the National Academy of Sciences, 112(35):10863-10868, 2015

12. Philipp Rothemund, Alar Ainla, Lee Belding, Daniel J Preston, Sarah Kurihara, Zhigang Suo, and George M Whitesides. A soft, bistable valve for autonomous control of soft actuators. Science Robotics, 3(16):eaar7986, 2018.

13. Yoël Forterre, Jan M Skotheim, Jacques Dumais, and Lakshminarayanan Mahadevan. How the venus flytrap snaps. Nature, 433(7024):421, 2005.

14. Anupam Pandey, Derek E Moulton, Dominic Vella, and Douglas $P$ Holmes. Dynamics of snapping beams and jumping poppers. EPL (Europhysics Letters), 105(2):24001, 2014.

15. Dominic Vella. Buffering by buckling as a route for elastic deformation. Nature Reviews Physics, page 1, 2019

16. Howon Lee, Chunguang Xia, and Nicholas X Fang. First jump of microgel; actuation speed enhancement by elastic instability. Soft Matter, 6(18):4342-4345, 2010.

17. A.N. Gent. A new constitutive relation for rubber. Rubber Chemistry Tech., 69:59-61, 1996

18. E. Riks. An incremental approach to the solution of buckling and snapping problems. Int. J. Solids Struct., 15:524-551, 1979.

19. M.A. Crisfield. A fast incremental/iterative solution procedure that handles snap-through. Computers and Structures, 13:55-62, 1983.

20. John W Hutchinson. Buckling of spherical shells revisited. Proceedings of the Royal Society A: Mathematical, Physical and Engineering Sciences, 472(2195):20160577, 2016.

21. Anna Lee, Francisco López Jiménez, Joel Marthelot, John W Hutchinson, and Pedro M Reis. The geometric role of precisely engineered imperfections on the critical buckling load of spherical elastic shells. Journal of Applied Mechanics, 83(11):111005, 2016.

22. R. Zoelly. Ueber ein knickungsproblem an der kugelschale. PhD thesis, ETH Zurich, 1915

23. Bastiaan Florijn, Corentin Coulais, and Martin van Hecke. Programmable mechanical metamaterials. Phys. Rev. Lett., 113:175503, Oct 2014. . URL https://link.aps.org/doi/10.1103/ PhysRevLett.113.175503.

24. Lucia Stein-Montalvo, Paul Costa, Matteo Pezzulla, and Douglas P. Holmes. Buckling of geometrically confined shells. Soft Matter, 15:1215-1222, 2019. . URL http://dx.doi.org/10. 1039/C8SM02035C.

25. Johannes T. B. Overvelde, David M. J. Dykstra, Rijk de Rooij, James Weaver, and Katia Bertoldi. Tensile instability in a thick elastic body. Phys. Rev. Lett., 117:094301, Aug 2016. . URL https://link.aps.org/doi/10.1103/PhysRevLett.117.094301.

26. Alan Needleman. Inflation of spherical rubber balloons. International Journal of Solids and Structures, 13(5):409-421, 1977.

27. Ingo Müller and Peter Strehlow. Rubber and rubber balloons: paradigms of thermodynamics, volume 637. Springer Science \& Business Media, 2004.

28. Michael Gomez, Derek E. Moulton, and Dominic Vella. Dynamics of viscoelastic snapthrough. Journal of the Mechanics and Physics of Solids, 124:781 - 813, 2019. ISSN 0022-5096. . URL http://www.sciencedirect.com/science/article/pii/S0022509618305131.

29. T.-X. Yu D. Karagiozova, X.-W. Zhang. Static and dynamic snap-through behaviour of an elastic spherical shell. Acta Mechanica Sinica, 28(3):695, 2012. . URL http://ams.cstam.org. $\mathrm{cn} /$ EN/abstract/article_143445.shtml.

30. Nikolaos Vasios, Andrew J Gross, Scott Soifer, Johannes TB Overvelde, and Katia Bertoldi. Harnessing viscous flow to simplify the actuation of fluidic soft robots. Soft robotics, 2019.

31. Benjamin Gorissen, Edoardo Milana, Arne Baeyens, Eva Broeders, Jeroen Christiaens, Klaas Collin, Dominiek Reynaerts, and Michael De Volder. Hardware sequencing of inflatable nonlinear actuators for autonomous soft robots. Advanced Materials, 31(3):1804598, 2019.

32. Lindsey Hines, Kirstin Petersen, and Metin Sitti. Inflated soft actuators with reversible stable deformations. Advanced Materials, 28(19):3690-3696, 2016.

33. Eran Ben-Haim, Lior Salem, Yizhar Or, and Amir D. Gat. Single-input control of multiple fluiddriven elastic actuators via interaction between bistability and viscosity. Soft Robotics, $0(0)$ : null, 2019.

34. Sanjoy Mahajan. The art of insight in science and engineering: mastering complexity. MIT Press, 2014.

35. Ahmad Rafsanjani, Yuerou Zhang, Bangyuan Liu, Shmuel M. Rubinstein, and Katia Bertoldi. Kirigami skins make a simple soft actuator crawl. Science Robotics, 3(15), 2018. . URL https://robotics.sciencemag.org/content/3/15/eaar7555.

36. Michael T. Tolley, Robert F. Shepherd, Bobak Mosadegh, Kevin C. Galloway, Michael Wehner, Michael Karpelson, Robert J. Wood, and George M. Whitesides. A resilient, untethered soft robot. Soft Robotics, 1(3):213-223, 2014. . URL https://doi.org/10.1089/soro.2014.0008.

37. Robin M Neville, Rainer MJ Groh, Alberto Pirrera, and Mark Schenk. Shape control for experimental continuation. Physical review letters, 120(25):254101, 2018.

38. Nakul Prabhakar Bende, Arthur A Evans, Sarah Innes-Gold, Luis A Marin, Itai Cohen, Ryan C Hayward, and Christian D Santangelo. Geometrically controlled snapping transitions in shells with curved creases. Proceedings of the National Academy of Sciences, 112(36):1117511180, 2015.

39. Jordan R Raney, Neel Nadkarni, Chiara Daraio, Dennis M Kochmann, Jennifer A Lewis, and Katia Bertoldi. Stable propagation of mechanical signals in soft media using stored elastic energy. Proceedings of the National Academy of Sciences, 113(35):9722-9727, 2016.

40. Neel Nadkarni, Andres F Arrieta, Christopher Chong, Dennis M Kochmann, and Chiara Daraio. Unidirectional transition waves in bistable lattices. Physical review letters, 116(24): 244501, 2016

41. Sicong Shan, Sung H Kang, Jordan R Raney, Pai Wang, Lichen Fang, Francisco Candido,
Jennifer A Lewis, and Katia Bertoldi. Multistable architected materials for trapping elastic strain energy. Advanced Materials, 27(29):4296-4301, 2015.

42. Tian Chen, Osama R Bilal, Kristina Shea, and Chiara Daraio. Harnessing bistability for directional propulsion of soft, untethered robots. Proceedings of the National Academy of Sciences, 115(22):5698-5702, 2018.

43. Ahmad Rafsanjani, Abdolhamid Akbarzadeh, and Damiano Pasini. Snapping mechanical metamaterials under tension. Advanced Materials, 27(39):5931-5935, 2015. 